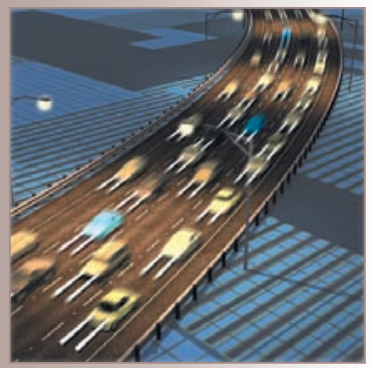

Editors: Frédéric Thiesse・frederic.thiesse@unisg.ch

Florian Michahelles·fmichahelles@ethz.ch

\title{
Perci: Pervasive Service Interaction with the Internet of Things
}

The advancement of ubiquitous computing technologies has greatly improved the availability of digital resources in the real world. Here, the authors investigate mobile interaction with tagged, everyday objects and associated information that's based on the Internet of Things and its technologies. Their framework for integrating Web services and mobile interaction with physical objects relies on information typing to increase interoperability. Two prototypes for mobile interaction with smart posters build upon this framework to realize multi-tag interaction with physical user interfaces. The authors' evaluation identifies usability issues regarding the design of physical mobile interactions, interfaces, and applications.

Gregor Broll, Massimo Paolucci, and Matthias Wagner DOCOMO Euro-Labs

\section{Enrico Rukzio}

Lancaster University

Albrecht Schmidt

University of Duisburg-Essen

Heinrich Hußmann

University of Munich
T he advancement of ubiquitous computing technologies, ${ }^{1}$ such as wireless networks and mobile devices, has greatly increased the availability of digital information and services in our daily lives and changed how we access and use them. Another technology that extends digital resources to the real world is the Internet of Things, ${ }^{2}$ which connects such resources with everyday objects by augmenting the latter with RFID or Near Field Communication (NFC) tags. ${ }^{3}$ This way, real-world objects get digital identities and can then be integrated into a network and associated with digital information or services. These objects can facilitate access to digital resources and support interaction with them - for example, through mobile devices that feature technologies for discovering, capturing, and using information from tagged objects.

Physical mobile interaction (PMI ${ }^{4,5}$ takes advantage of mobile devices that physically interact with tagged objects to facilitate interaction with associated information and services. Simply by touching or pointing at objects, users can interact with them - for example, touching NFC tags or taking pictures of visual markers with their mobile phones. Due to its increased simplicity and directness, physical interaction can make mobile interaction with "people, places, and things" ${ }^{6}$ more convenient and intuitive. Tagged objects can serve as physical user interfaces 


\section{Pervasive Service Interaction with the Internet of Things}

that advertise ubiquitous information, facilitate the information's discovery, complement mobile interfaces, and adopt some of those interfaces' features.

An increasing number of applications are adopting mobile interaction with tagged objects for service discovery and invocation, information retrieval, ticketing, or mobile payment. Tagging technologies' advancement and increasing dissemination have facilitated a movement from the original, simple interactions with single tags to more complex multitag interaction (MTI $)^{7,8}$ with multiple tags and objects (Figure 1), shifting interaction's focus from mobile devices to tagged objects serving as physical user interfaces, respectively.

To leverage mobile interaction with physical objects and digital resources from, for example, the Internet of Things, the Pervasive Service Interaction (Perci) project (see www.hcilab.org/ projects/perci) has examined different aspects of PMI. It has investigated basic mechanisms for integrating PMI with mobile services, improving interoperability between them, developing PMI toward MTI, and improving the usability of physical user interfaces and interaction techniques. Here, we look at Perci's main contributions to mobile interaction with the Internet of Things, focusing on the implementation, design, and usability of physical mobile interactions and applications.

\section{The Perci Framework}

The University of Munich and DOCOMO EuroLabs developed the Perci framework ${ }^{5}$ to implement basic mechanisms for seamless interaction between mobile services and tagged objects. On one hand, Perci uses service descriptions to automatically generate interfaces that support mobile interaction with tagged objects. On the other, the framework relies on PMI to facilitate access to services and interaction with them.

\section{Architecture}

The Perci architecture is divided into three main parts (Figure 2): mobile devices interact with tagged physical objects that are associated with Web services and provide information for their invocation - for example, a poster for a movieticketing service. The generic universal client on mobile devices comprises modules to handle the interaction with tagged objects (interaction client) and associated services (service client).

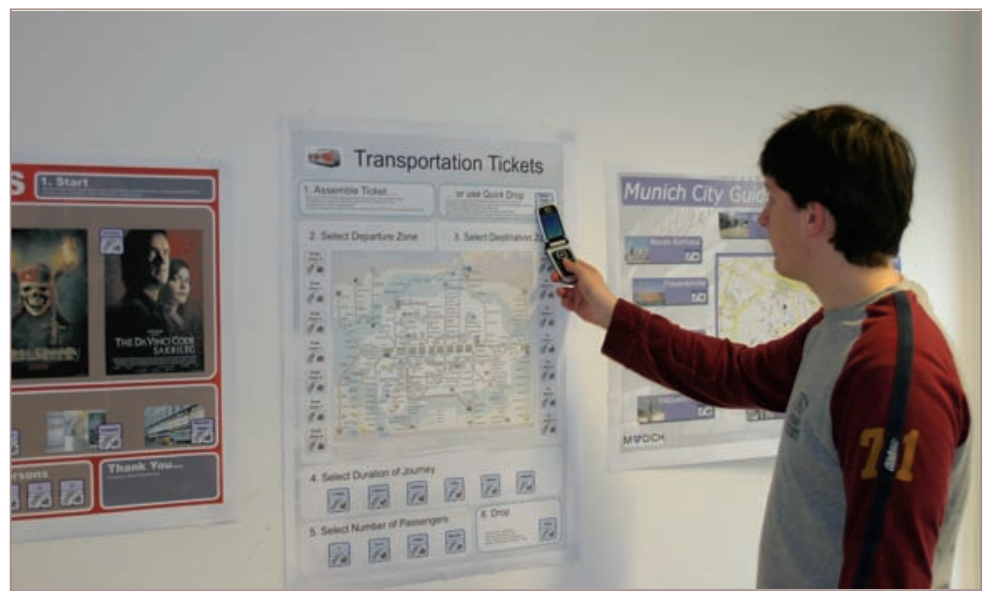

Figure I. Physical mobile interaction (PMI) with multiple tags and objects. Tagged objects can serve as physical user interfaces that facilitate interaction with associated information and services. (Research prototypes include material from www.actuacine.net and www.mvg-mobil.de.)

It's connected to the interaction proxy, which manages the communication between Web services and mobile clients while keeping them independent from each other. This proxy generates interfaces from service descriptions to support PMI on mobile devices and manages the interaction with services on the universal client's behalf.

\section{Semantic Integration}

One mechanism for integrating Web services and PMI is to automatically generate interfaces that support this interaction. For this purpose, the Perci framework extends the Semantic Markup for Web Services (OWL-S) descriptions with its custom Service User Interface Annotation (SUIA) ontology. This ontology complements the basic description of parameters in OWL-S with additional information for rendering mobile user interface widgets. More precisely, the Abstract Widget Type Model specifies common interface widgets in a device-independent way and suggests suitable widgets for each service parameter. These widgets will help users acquire information from tagged objects and thus provide input for the service parameters they represent.

The framework uses abstract parameter types to correctly map information from tagged objects to service parameters. This generic typing model adds semantic meaning to both, defıning them as specific types with individual properties. Tagged objects are associated with specific services (for example, a poster for buying movie tickets 


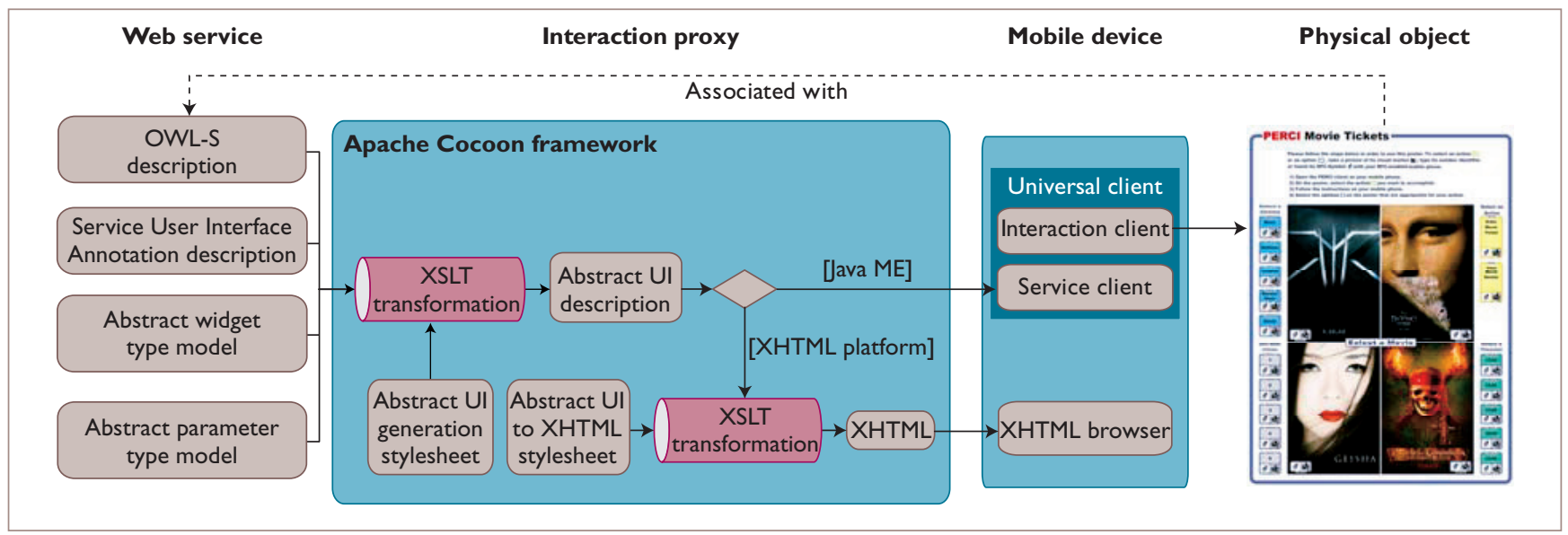

Figure 2. The Pervasive Service Interaction (Perci) framework architecture. Perci provides for integrated interaction between mobile services and tagged objects.

via a ticketing service). Similarly, information on these objects (such as options on a poster) is associated with the services' input parameters. Using the same typing model for service parameters and information from tagged objects lets applications allocate them to one another correctly and dynamically. This is essential for enabling interoperability between information and services, especially across different objects, and prevents a static one-to-one mapping.

\section{Dynamic Mobile Interfaces}

Service descriptions and extensions provide the basis for dynamically generating mobile interfaces in the Perci framework. The interaction proxy uses the XML-publishing framework Cocoon (http://cocoon.apache.org) to derive an abstract UI description from this information according to transformation rules from an XSLT stylesheet (Figure 2). This compact description contains all information for rendering and customizing mobile interfaces. The interaction proxy uses Cocoon's multichannel publishing to generate different (mobile) user interfaces from the same abstract UI description. The prototype either applies another XSLT transformation to generate an XHTML interface for (mobile) Web browsers or returns the description to a Java ME application that renders the user interface according to its own rules (Figure 2). Mobile clients support PMI according to their individual technical configurations - for example, manual input in HTML forms or the recognition of visual markers or NFC tags on Java ME-enabled phones.

Mobile Interaction with Smart Posters

To demonstrate mobile interaction with services through physical interaction with tagged objects, we developed a prototype for mobile ticketing that implements the universal client as a Java ME application on top of the Perci framework. ${ }^{5}$

\section{Physical Interaction Design}

The prototype includes two posters that are associated with Web services for mobile ticketing (movies and transportation) and provide several options (movies, cinemas, stations, numbers of passengers, and so on) for invoking those services (Figure 3a). We tagged each option with a numeric identifier, an NFC tag, and a visual marker (Figure 3b). These tags contain or reference the information that the option represents, such as the name of a cinema. The mobile client supports the PMI techniques touching (using NFC; Figure 3c), pointing (recognizing visual markers; Figure 3d), and direct input of numeric identifiers (Figure 3e). Users can employ these techniques to interact with the tagged options on the poster, acquire information, and use that data to invoke associated services.

\section{Interaction with Mobile Services}

The prototype distinguishes between action tags that contain services' URLs and parameter tags that contain information for those services' invocation. Users initiate an interaction with a service by selecting its action tag on a poster. Their mobile-client application forwards the acquired URL to the interaction proxy, which retrieves the abstract UI description from the service. Next, the mobile client uses this description to render a user interface with widgets for each service parameter accord- 


\section{Related Work in Physical Mobile Interaction}

n 1999, Roy Want and his colleagues presented some of the first examples for linking everyday objects (such as books, documents, and business cards) with digital resources (such as electronic documents, URLs, and email addresses) through RFID tags.' Since then, ubiquitous computing applications have used various technologies to implement the tagging of and interaction with physical objects, including Near Field Communication (NFC), ${ }^{2}$ Bluetooth, infrared beacons, ${ }^{3}$ laser pointers, ${ }^{4}$ GPS, or the recognition of visual markers, ${ }^{5}$ which will eventually be displaced by markerless image recognition. ${ }^{6}$

Researchers have built different interaction techniques and metaphors on top of these enabling technologies to make their use more familiar and intuitive. For example, Enrico Rukzio and his colleagues ${ }^{4}$ use the techniques touching (using NFC), pointing (using a laser pointer), and scanning (using Bluetooth) for interaction with smart-home appliances. Physical hyperlinks use the simple interaction with single NFC/RFID tags as shortcuts for more complex actions, ${ }^{7}$ such as setting up wireless connections. Hovering visualizes tags' content before using them. ${ }^{8}$

Although these examples show single-tag interactions' simplicity, others rely on more complex interactions with multiple tags. In one study, the authors present a framework for requesting services by touching RFID tags with different functionalities ${ }^{9}$ — for example, object identification or printing. Derek Reilly and his colleagues have augmented maps with RFID tags, turning them into interactive surfaces that support path-, lasso-, or menu-select with mobile devices. ${ }^{10}$ Robert Hardy and his colleagues have extended this static physical interface to a dynamic NFC display that projects an application interface onto a grid of NFC tags." Users manipulate the projected interface by touching the tags with NFC-enabled mobile devices.

The development of physical mobile interaction (PMI) met up with the Internet of Things, ${ }^{12}$ whose infrastructure uses RFID tags to attach a unique Electronic Product Code (EPC) to objects to identify them on a network for monitoring, for example. PMI complements the Internet of Things because it relies on similar basic technologies. The research done in the
Pervasive Service Interaction (Perci) project we describe in the main text is one of the first approaches to build on the integration of the technologies just presented to facilitate user interaction with mobile services and tagged, physical objects from the Internet of Things.

\section{References}

I. R. Want et al., "Bridging Physical and Virtual Worlds with Electronic Tags," Proc. SIGCHI Conf. Human Factors in Computing Systems: The CHI Is the Limit (CHI 99), ACM Press, 1999, pp. 370-377.

2. R. Want, "An Introduction to RFID Technology," IEEE Pervasive Computing, vol. 5, no. I, 2006, p. 25.

3. T. Kindberg et al., "People, Places, Things: Web Presence for the Real World," Mobile Network Applications, vol. 7, no. 5, 2002, pp. 365-376.

4. E. Rukzio et al., "An Experimental Comparison of Physical Mobile Interaction Techniques: Touching, Pointing, and Scanning," Proc. 8th Int'l Conf. Ubiquitous Computing (Ubicomp 06), LNCS, Springer-Verlag, 2006, pp. 87-104

5. M. Rohs and B. Gfeller, "Using Camera-Equipped Mobile Phones for Interacting with Real-World Objects," Advances in Pervasive Computing, Austrian Computer Society (OCG), 2004, pp. 265-27I.

6. T. Quack, H. Bay, and L.J. Van Gool, "Object Recognition for the Internet of Things," Proc. Internet of Things Conf. (IOT 08), 2008, pp. 230-246.

7. J. Schwieren and G. Vossen, "Implementing Physical Hyperlinks for Mobile Applications using RFID Tags," Proc. I/th Int'l Database Eng. and Applications Symp. (IDEAS 07), IEEE CS Press, 2007, pP. 154-162.

8. P. Välkkynen, "Hovering: Visualising RFID Hyperlinks in a Mobile Phone," Proc. Mobile Interaction with the Real World, 2006, pp. 27-29.

9. J. Riekki, T. Salminen, and I. Alakärppä, "Requesting Pervasive Services by Touching RFID Tags," IEEE Pervasive Computing, vol. 5, no. I, 2006, pP. 40-46.

10. D. Reilly et al., "Just Point and Click?: Using Handhelds to Interact with Paper Maps," Proc. 7th Int'l Conf. Human-Computer Interaction with Mobile Devices and Services (MobileHCl 05), vol. 3, ACM Press, 2005, pp. 239-242.

II. R. Hardy and E. Rukzio, "Touch \& Interact: Touch-Based Interaction of Mobile Phones with Displays," Proc. 10th Int'l Conf. Human-Computer Interaction with Mobile Devices and Services (MobileHCl 08), ACM Press, 2008, pp. 245-254.

12. ITU Internet Reports 2005: The Internet of Things, 7th ed., ITU, 2005; www.itu. int/osg/spu/publications/internetofthings/. ing to the service parameters' abstract widget types. Finally, the user can arbitrarily interact with the parameter tags on the poster and use the UI widgets to collect those tags' information for further interaction with the service.

The prototype uses the abstract parameter types to correctly allocate information from parameter tags to service parameters. Mobile services use the typing model to specify the types of parameter values they need to be invoked, and parameter tags on physical objects specify the type of information they can provide for that purpose. This way, the common use of a global typing model improves interoperability between information and services. PMI supports these interactions by breaking them down to the simple interaction with a few tags.

\section{Evaluation}

We conducted a qualitative user study with 17 subjects (13 male, four female, average age 29) to evaluate the prototype's interaction design and compare its techniques for PMI. ${ }^{9}$ All subjects accomplished the same task - ordering a ticket via the movie poster - using these tech- 


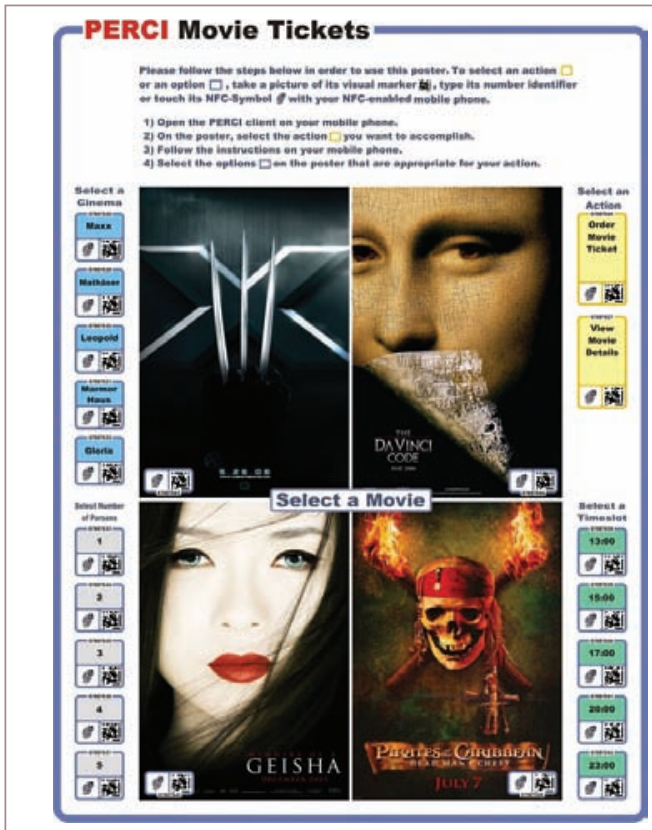

(a)

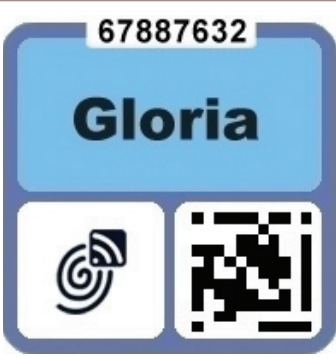

(b)

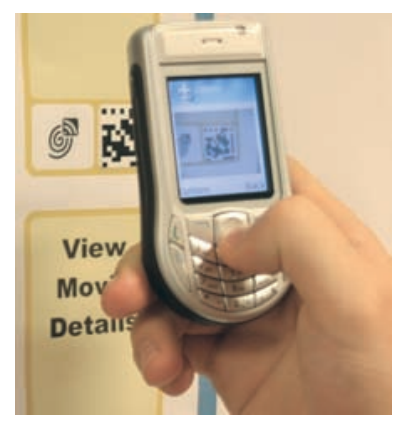

(d)

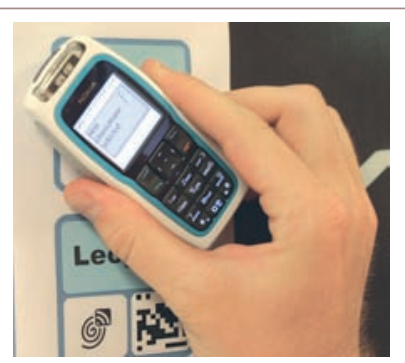

(c)

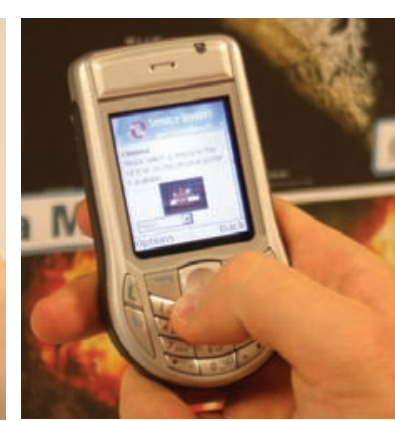

(e)

Figure 3. Poster for mobile ticketing. The (a) poster contains (b) tagged options that support the interaction techniques (c) touching, (d) pointing, and (e) direct input of numeric identifiers.

niques in alternate order. We tested touching and pointing with a Java ME application and direct input with a mobile HTML browser to cover all supported client platforms.

The subjects evaluated the interaction techniques with questionnaires and used a Likert scale to assess to what degree different properties applied to them completely (4) or not at all (0). Touching received the best ratings for handling (average value: 3.4), enjoyment (2.7), and innovation (3.0), and was slightly less reliable than direct input (3.5 versus 3.7). Pointing got the worst ratings for handling (0.9) and reliability (1.7) due to the tedious interaction with the phonecam. Nevertheless, subjects regarded it as more innovative (2.5) and enjoyable (1.7) than direct input, which they saw as very reliable (3.7) and easy to handle (2.9) but neither innovative (0.8) nor enjoyable (0.9).

At first, many subjects had problems with the general interaction design: they didn't know how to start the interaction, were confused by the lack of a predefined interaction sequence, couldn't think of a possible workflow, or didn't understand the distinction between action and parameter tags without an explanation. These problems were due most likely to the novelty of the interaction, and the subjects usually resolved them quickly. The study also showed that subjects often ignored instructions on the posters.

\section{Collect\&Drop}

The first prototype showed user interaction with physical objects and mobile services on a basic level but was restricted to simple interactions. To leverage MTI and better exploit the interoperability between services and information, CollectEDrop ${ }^{7}$ builds on the first prototype to realize a more flexible approach to their combination and reuse across different objects and services.

\section{Leveraging Multi-Tag Interaction}

As a prerequisite for more flexible MTI, and unlike the first prototype, CollectctDrop doesn't rigidly map actions and parameters to dedicated tags but instead maps them to self-contained information items that adhere to the abstract parameter types. Action items describe services and the information needed for their execution, including such information's abstract parameter types. Similarly, data items contain parameter information for service execution.

Separating actions and parameters on the level of information items has several advantages. Information items can be more independent, which supports their reuse and combination. This means that we can invoke a service from an action item with any data item from any object, as long as the two items' abstract parameter types match. Likewise, we 


\section{Pervasive Service Interaction with the Internet of Things}

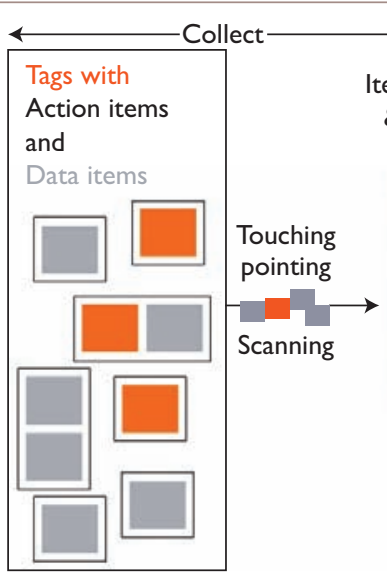

(a)

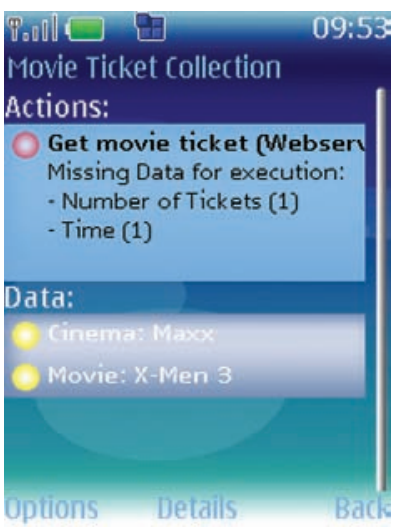

(b)

Figure 4. Collect\&Drop's interaction workflow. We can see (a) the workflow for collecting information items from tagged objects and (b) storing them in collections and using them to invoke mobile services.

can reuse data items with different services from different objects. This way, CollectctDrop can better exploit the interoperability between information and services and realize MTI across different objects. Decoupling tags and information also enables us to combine action items and data items on the same tag (hybrid tags).

\section{Collect\&Drop's Interaction Design}

Collect\&Drop reimplements the universal client with a focus on MTI. It builds on the Perci framework and the first prototype, inherits its interaction techniques, services, and posters, and adds a new one for sightseeing with tagged points of interest (Figure 1).

Figure 4a shows CollectetDrop's interaction workflow. During the collect phase, mobile devices interact with tags (black frames) on physical objects to acquire their data or action items (blue and orange squares). Each tag can contain multiple information items, and Collect\&tDrop uses data containers, or collections, to store and manage these items on mobile devices (Figure 4b). It creates a new collection for each action item and adds suitable data items according to their abstract parameter types. This mechanism implements the matching between action items whose services require certain types of information for invocation and data items that can provide that information. During the drop phase, CollectCtDrop uses data items to invoke the service an action item specifies (Figure 4a). Services can return data items as a result of being invoked (for example, a cinema ticket), which other services can use.

The separation of information items, their interoperability based on abstract parameter types, and their storage support new features for MTI. The posters for transportation and sightseeing map their action items to dedicated drop tags to trigger interaction with their services explicitly. On the other hand, the movieticketing poster uses hybrid tags for a less complex interaction design because users collect the action item for the service implicitly with any other tag. Collect\&Drop also supports cross-object interaction because data items for cinemas and sights on the posters for movie ticketing and sightseeing also comprise location information that users can utilize with the drop tag on the transportation poster.

\section{Evaluation}

We evaluated Collect\&tDrop in a qualitative user study with 15 subjects (10 male, five female, average age 25.5) that focused on its interaction design and features for MTI. ${ }^{7}$ The subjects carried out different tasks to evaluate the interaction with each poster, its features, and cross-object interaction between posters. They assessed Collect\&Drop with questionnaires that used a Likert scale to rate different properties regarding its design and features from worst (1) to best (5).

Collect\&Drop was well received, and subjects regarded its concept as very comprehensible (average value: 4.7 ), the client application as easy to use (4.3), and the collections as very useful (4.5). Nevertheless, some subjects had problems starting the interaction, which they regarded as unfamiliar and confusing at first, but which became more intuitive after a while. 
Eight subjects were aware of the differences between posters with hybrid tags and dedicated drop tags. A comparison showed that subjects found hybrid tags to be easier (4.7 versus 4.2), much faster (4.9 versus 3.4), and more intuitive (4.5 versus 3.7 ) to use than drop tags.

To test cross-object interaction, the subjects had to submit a data item from one poster (a movie ticket from the ticketing service) to an action item on another (the drop tag on the transportation poster). They regarded cross-object interaction as very quick (4.8) and easy to use (4.2), comprehensible (3.9), and fairly intuitive (3.3). Subjects viewed the posters as independent use cases that were closely related to their services. Many subjects stated that the interoperability between them seemed to be strange at first but quickly became more comprehensible.

We numbered the option categories on the ticketing posters to guide the users' interaction with them. Although four subjects didn't follow this numbering, all subjects approved the numbering and a given order of interaction, which they described as intuitive, error-preventing, and helpful for beginners. Nevertheless, they felt such numbering shouldn't be mandatory and that the posters should allow interaction with tags in arbitrary order.

The evaluation of the poster-numbering complements observations about initial problems with PMI. Further work on this topic ${ }^{10}$ shows how different cues on physical interfaces can reduce initial inhibition thresholds of PMI and increase its learnability.

$\mathrm{T}$ he Perci project's results can serve as lessons learned and encourage further research into PMI and MTI. More thorough evaluations of their technical aspects and usability will be needed to confirm the usefulness of MTI and its features. Our current results only hint at the impact of physical user interface design on overall interaction design. Future work will show how the Internet of Things and PMI can benefit from further integration.

\section{Acknowledgments}

Perci (www.hcilab.org/projects/perci) is a bilateral project between the University of Munich and DOCOMO Euro-Labs and was funded by the latter. We thank Sven Siorpaes, Markus Haarländer, and Kevin Wiesner for their contributions.

\section{References}

1. M. Weiser, "The Computer for the 21st Century," Human-Computer Interaction: Toward the Year 2000, R.M. Baecker et al., eds., Morgan Kaufmann, 1995, pp. 933-940.

2. ITU Internet Reports 2005: The Internet of Things, 7th ed., ITU, 2005; www.itu.int/osg/spu/publications/ internetofthings/.

3. R. Want, "An Introduction to RFID Technology," IEEE Pervasive Computing, vol. 5, no. 1, 2006, p. 25.

4. E. Rukzio et al., "An Experimental Comparison of Physical Mobile Interaction Techniques: Touching, Pointing, and Scanning," Proc. 8th Int'l Conf. Ubiquitous Computing (Ubicomp 06), LNCS, Springer-Verlag, 2006, pp. 87-104.

5. G. Broll et al., "Supporting Mobile Service Usage through Physical Mobile Interaction," Proc. 5th IEEE Int'l Conf. Pervasive Computing and Communications (PERCOM 07), IEEE CS Press, 2007, pp. 262-271.

6. T. Kindberg et al., "People, Places, Things: Web Presence for the Real World," Mobile Network Applications, vol. 7, no. 5, 2002, pp. 365-376.

7. G. Broll et al., "Collect\&Drop: A Technique for MultiTag Interaction with Real World Objects and Information," Proc. European Conf. Ambient Intelligence (AmI 08), E. Aarts et al., eds., LNCS 5355, Springer-Verlag, 2008, pp. 175-191.

8. R. Hardy and E. Rukzio, "Touch \& Interact: TouchBased Interaction of Mobile Phones with Displays," Proc. 10th Int'l Conf. Human-Computer Interaction with Mobile Devices and Services (MobileHCI 08), ACM Press, 2008, pp. 245-254.

9. G. Broll et al., "Comparing Techniques for Mobile Interaction with Objects from the Real World," Proc. 3rd Int'l Workshop on Pervasive Mobile Interaction Devices (Permid 07), 2007; www.permid.org/2007/pdf/ proceedings_permid2007.

10. G. Broll et al., "Improving the Accessibility of NFC/ RFID-Based Mobile Interaction through Learnability and Guidance," Proc. 11th Int'l Conf. Human-Computer Interaction with Mobile Devices and Services (MobileHCI 09), ACM Press, 2009, pp. 1-10.

Gregor Broll is a PhD candidate at DOCOMO Euro-Labs. His current research focuses on the design and usability of applications and physical user interfaces for mobile interaction with Near Field Communication. Broll has an MSc in media informatics from the University of Munich (Ludwig-Maximilians-Universitaet), where he's been working on mobile service interaction as a research assistant and $\mathrm{PhD}$ student. Contact him at broll@docomolab-euro.com. 


\section{Pervasive Service Interaction with the Internet of Things}

Massimo Paolucci is a senior researcher at D0C0MO EuroLabs. His research interests include discovery and composition of Semantic Web services with a specific focus on mobile and ubiquitous computing. Paolucci has an MSc in computational linguistics from Carnegie Mellon University, an MSc in intelligent systems from the University of Pittsburgh, and a Laurea from Universita' di Milano. He's a member of the OWL-S coalition. Contact him at paolucci@docomolab-euro.com.

Matthias Wagner is a manager of the Smart and Secure Services Research Group at DOCOMO Euro-Labs. His main research interests are in service provisioning and contextual intelligence for the next generation of mobile systems. Wagner has an MSc and PhD in computer science. He's a member of the ACM. Contact him atwagner@docomolab-euro.com.

Enrico Rukzio is an RCUK academic fellow and lecturer in mobile human-computer interaction in the computing department at Lancaster University. His research focuses on mobile interactions with pervasive user interfaces, in particular touch-based mobile interaction with vertical and horizontal interactive surfaces, interactions with personal projections, and mobile navigation. Rukzio has an MSc in computer science from the Technical University of Dresden and a PhD from the University of Munich. He's the founder of the Pervasive Mobile Interaction Devices (PERMID) and Mobile Interaction with the Real Word (MIRW) workshop series. Contact him at rukzio@comp.lancs.ac.uk.

Albrecht Schmidt is a professor of pervasive computing and user interface engineering at the University of Duisburg-Essen in Germany. His teaching and research interests are in media informatics and ubiquitous computing, in particular, the area of user interface engineering. Schmidt has a PhD from Lancaster University. Contact him at albrecht.schmidt@icb.uni-due.de.

Heinrich Hußmann is a full professor of computer science at the University of Munich (Ludwig-MaximiliansUniversitaet), Germany. He's interested in the application of IT to communication media and in usability aspects of innovative user interfaces. Hußmann has a PhD from Passau University and a habilitation degree from Munich University of Technology. He's a member of the ACM and the IEEE Computer Society. Contact him at hussmann@ifi.lmu.de.

Selected CS articles and columns are also available

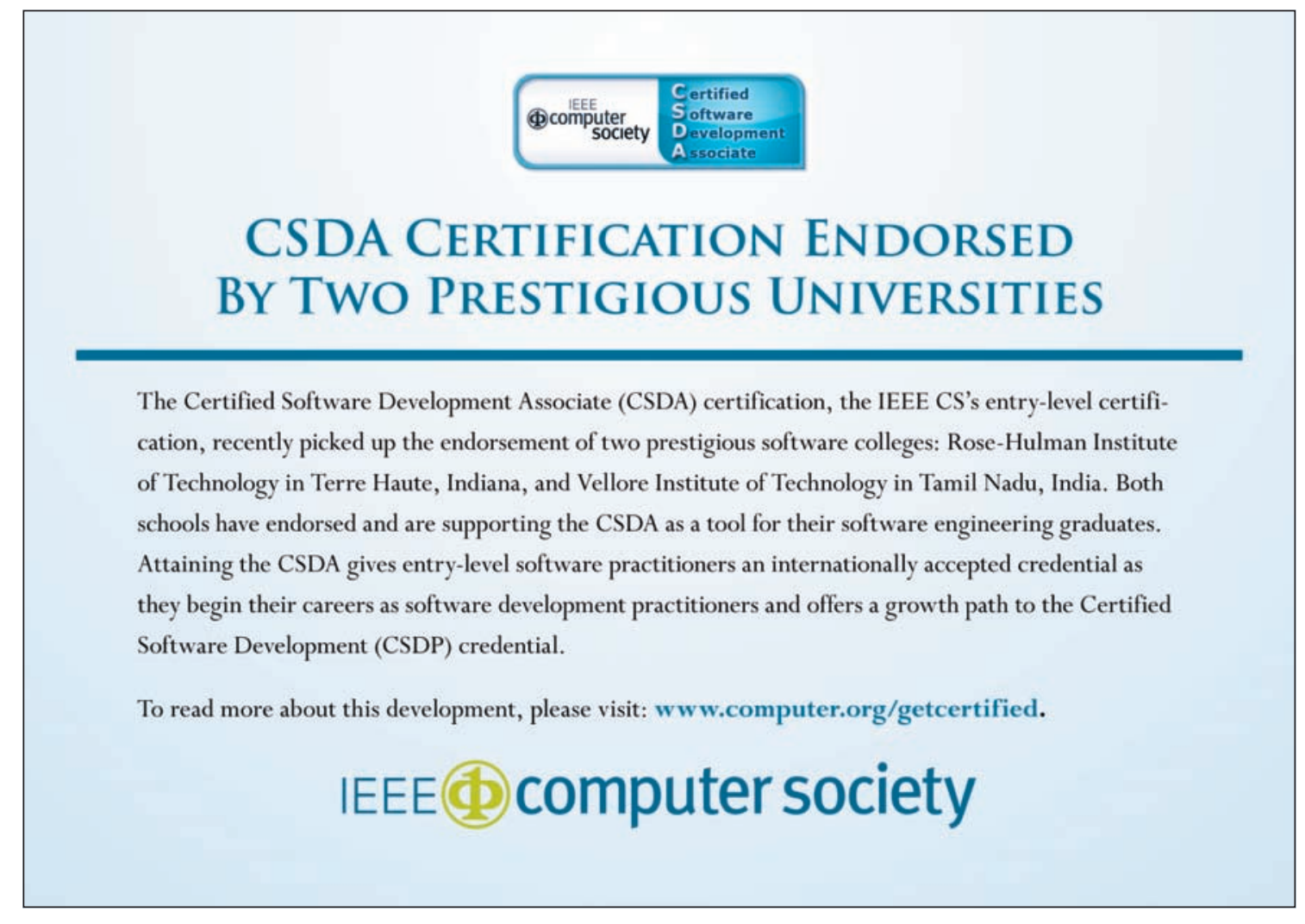


This article was featured in

\section{computing now}

For access to more content from the IEEE Computer Society, see computingnow.computer.org.

- IEEE

Celebrating 125 Years of Engineering the Future

\section{IEEE(D) Computer society}

\section{Top articles, podcasts, and more.}
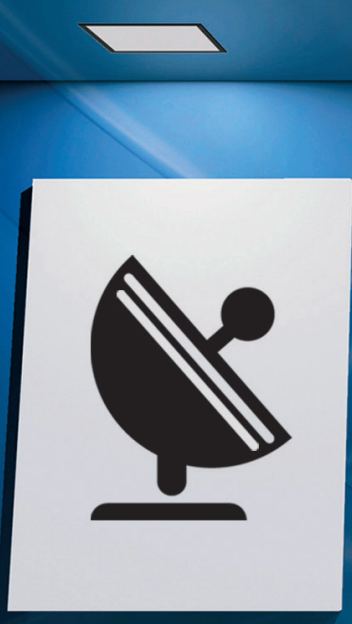
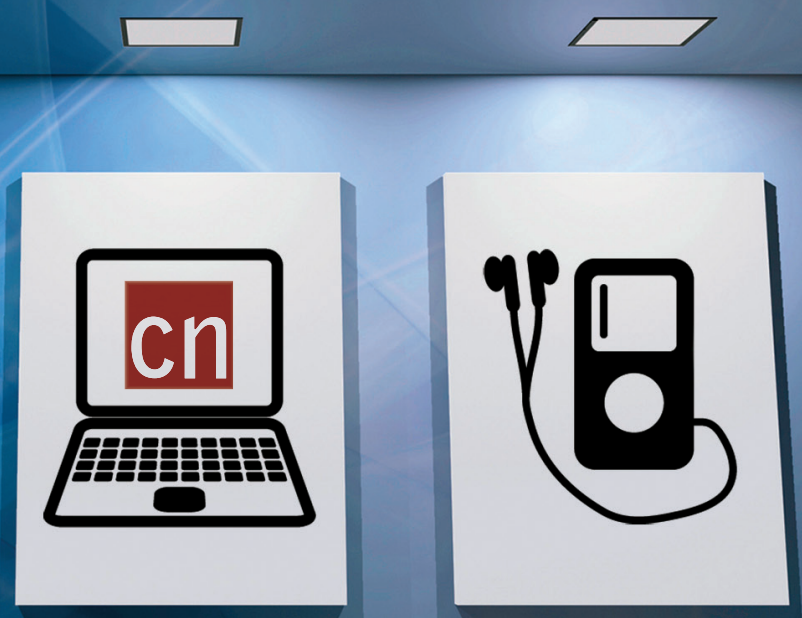